\title{
Computer Digital Technology-Based Educational Platform for Protection and Activation Design of Ming Furniture
}

\author{
Zhe Peng ${ }^{1}{ }^{1}$ and Yichen $D u^{2}$ \\ ${ }^{1}$ The School of Design, China Academy of Art, Hangzhou, 310012 Zhejiang, China \\ ${ }^{2}$ Experimental Teaching Department, China Academy of Art, Hangzhou, 310012 Zhejiang, China \\ Correspondence should be addressed to Zhe Peng; 0103034@caa.edu.cn
}

Received 30 October 2021; Revised 22 November 2021; Accepted 30 November 2021; Published 17 December 2021

Academic Editor: Mu-Yen Chen

Copyright (c) 2021 Zhe Peng and Yichen Du. This is an open access article distributed under the Creative Commons Attribution License, which permits unrestricted use, distribution, and reproduction in any medium, provided the original work is properly cited.

\begin{abstract}
Chinese Ming-style furniture is the first of the three major furnitures in the world, which has high artistic value and complete structural system. The protection of Ming-style furniture and making its design show vitality that has always been a research topic in China and even in the world. Based on 3D virtual simulation technology, this paper uses 3D scanning reverse data acquisition technology and intelligent operation of computer engine to realize big data simulation and develops the design software of Ming furniture. By means of computer information technology, we interpret and present the structural thinking and design concept of Ming furniture and transform it into design program software. This has formed a system-wide educational software operation platform for knowledge reserve, thinking training, design and application, and achievement transformation. By applying the mechanism strategy of production and teaching to the talent training plan of colleges and universities, the underlying logic of talent training, technical management, and production management that can open up the innovative industry of Ming furniture is also constructed. After experimenting with the platform software, students are able to understand the relationship between the structure and form of Ming furniture and can design new styles that fit the logic of Ming furniture shapes as they prefer.
\end{abstract}

\section{Introduction}

Chinese Ming furniture is one of the top three pieces of furniture in the world, with high artistic value and a complete construction system. The conservation and design revitalization of Ming furniture has been a research topic in China and the world. There is a need to develop learning software for Ming-style furniture by relying on Chinese cutting-edge computer soft-computer and Internet technologies. Applying the software to the teaching experiment platform of the national higher education institutions will become an important means and motivation for the protection of cultural heritage of classical furniture and technological innovation of related cultural and creative industries. At the same time, this is also an important research direction for production and teaching. This paper introduces and elaborates on the platform construction, development ideas, technical support, and innovations of this software, thus forming a system-wide educational software operation platform of knowledge reserve, thinking training, design application, and result transformation. In a practical sense, it also builds the underlying logic that can open up the talent training, technology management, and production management of the Ming furniture innovation industry.

\section{The Platform Construction}

Ming furniture construction is the unity of science and technology, a perfect combination of practicality and aesthetics, the key content and key to the study of classical Chinese furniture [1]. Frontier technology development and application have promoted technological innovation in cultural heritage conservation and related cultural and creative industries and become an important research direction for industryacademia research [2]; so, educational software development based on digital technology for the conservation and 
revitalization design of Ming-style furniture is of great practical significance [3]. The educational experiment platform is divided into three sections: (1) learning in virtual exhibition hall, (2) construct knowledge learning, (3) innovative design experiment, and (4) model output (Figure 1).

Learning in virtual exhibition hall: through 20 pieces of digital reconstruction virtual simulation models of classical furniture, we can observe and learn the shape and structure of traditional furniture. Using 3D modeling and dynamic design, it supports the observation from the first perspective and realizes the observation experience of classic furniture entities handed down from ancient times, and the traditional teaching cannot realize the observation of the internal structure of classic furniture. At the same time, it supports the explosive structure decomposition and aggregation of the whole furniture. Structural symbols are the main constituent elements of the Ming furniture modeling symbol system, which mainly includes two symbol types: mortise and tenon and component [4]; so, the system will support the information prompt of each furniture component and mortise and tenon structure. This truly realizes the teaching resources and digital protection of virtual simulation (Figure 2).

Construct knowledge learning: mortise and tenon construction is the best part of traditional Chinese furniture and is the earliest description of scientific design in China [5]. Ming-style furniture is the pinnacle of the development of mortise and tenon technology [6]. It is divided into two parts: component recognition and whole device recognition. The modeling principle of Ming-style furniture lies in the relationship between the structure and form of the main components; that is, the structural mode of the core plate forms the basic form: (1) interactive learning of component recognition. Through interactive software operation, we can clearly and intuitively understand and identify the names of various components and the operation rules of mortise and tenon: (2) interactive experiment of whole device recognition. Through interactive software, the names and loading sequence of components and mortise and tenon nodes of the whole device are recognized. Thus, students can understand the key elements of modeling, that is, component modules and construction methods (Figure 3).

Innovative design experiment: according to the computer "Ming-style furniture structure module system," students enter the preset chair basic knot module system, design and reorganize according to the preset basic structure module and selection sequence, and design a new shape model based on Ming-style furniture knot law.

Output of renderings and models: the innovative furniture form system automatically generates $3 \mathrm{D}$ renderings and model data, which is compatible with $3 \mathrm{D}$ printing equipment and outputs proportional solid models. And the system can automatically generate the corresponding mortise and tenon structure of the 3D file, from the design drawings to the object of seamless transformation.

\section{Development Ideas}

Since the mortise and tenon structure is relatively stable and has the characteristics of standardized design, the parametric design work is also feasible [1]. The development of Ming furniture design software is based on the principle of the relationship between the shape and structure of Ming furniture itself, refines a form design thinking method which conforms to the existing classical shape structure law, and then transforms it into a computer virtual simulation digital module through digital acquisition. And based on the specific design algorithm, arrange reasonable experimental steps and automatically generate digital innovation results. The function of the parametric design of Ming furniture components is to realize the modular programming of the characteristic relations of furniture components with general standardization characteristics in order to achieve the function of computer-aided design [7].

3.1. Principle of Software Development. The principle of design thinking method is the unified relationship between furniture structure and shape. The reason why Ming furniture became Ming furniture system is the clue of its structure and form development. This clue is based on the orderly construction of several core structural modules, and the shape of each furniture is based on the combination and arrangement of several structural modules. Mastering the logical relationship between Ming furniture form and knot module is very important to identify the core components of a piece of furniture and can quickly interpret which components a whole piece can be decomposed into. On the contrary, designing a piece of Ming furniture is an orderly construction of core components, which is the principle of computer software development [8].

3.2. Principle of Computer Software Experiment. The principle of "interactive innovative design" is divided into four parts: preset core knot module, orderly construction, generation of mortise and tenon nodes, and renderings and generation of 3D models.

Preset the core knot module: based on chairs, we have a deep understanding of Ming furniture. 1000 pieces of chairs furniture samples are divided into 7 basic modules by summarizing the core modules. Each module extracts a number of corresponding typical forms, which become preset options.

Build in an orderly manner: each furniture core module corresponds to the furniture components. In the assembly of furniture, it is required to build in an orderly manner, which is interlocking. Only in this way can a stable frame system be formed. The computer sets the correct shape selection steps to conform to the assembly principle of each piece of furniture, which is the key to design a reasonable supporting frame [9]. At each step of the student's choice, the computer will calculate the corresponding result and show it as an effect, and at the final completion, the computer will present the calculated result as a 360 -degree 3D effect that can be operated in a circular view.

Generate mortise and tenon nodes: the core components of each piece of furniture will form important mortise and tenon nodes, such as the seat surface of a chair, and there will be mortise and tenon nodes with "lattice corners, edges and grooved panels." The opening mode of mortise and 


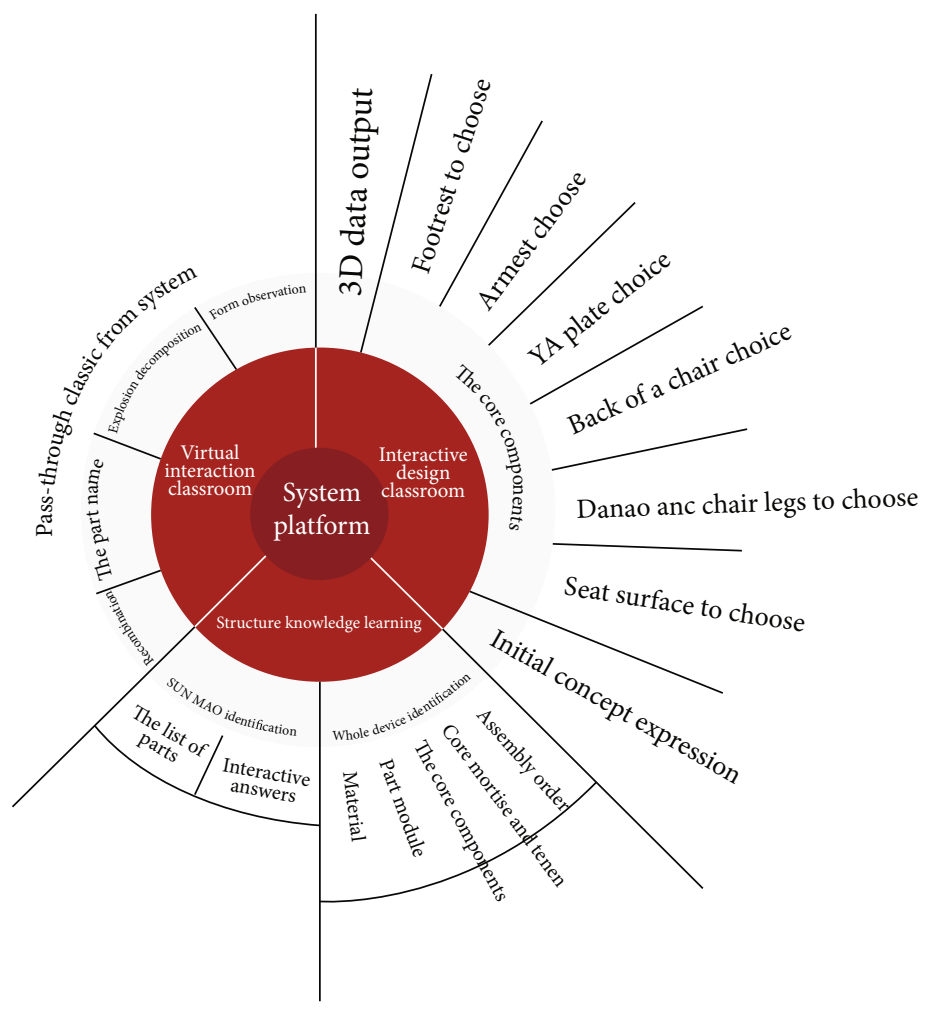

Ming-style furniture protection and activation virtual simulation experiment

FIGURE 1: The experiment platform mind mapping.

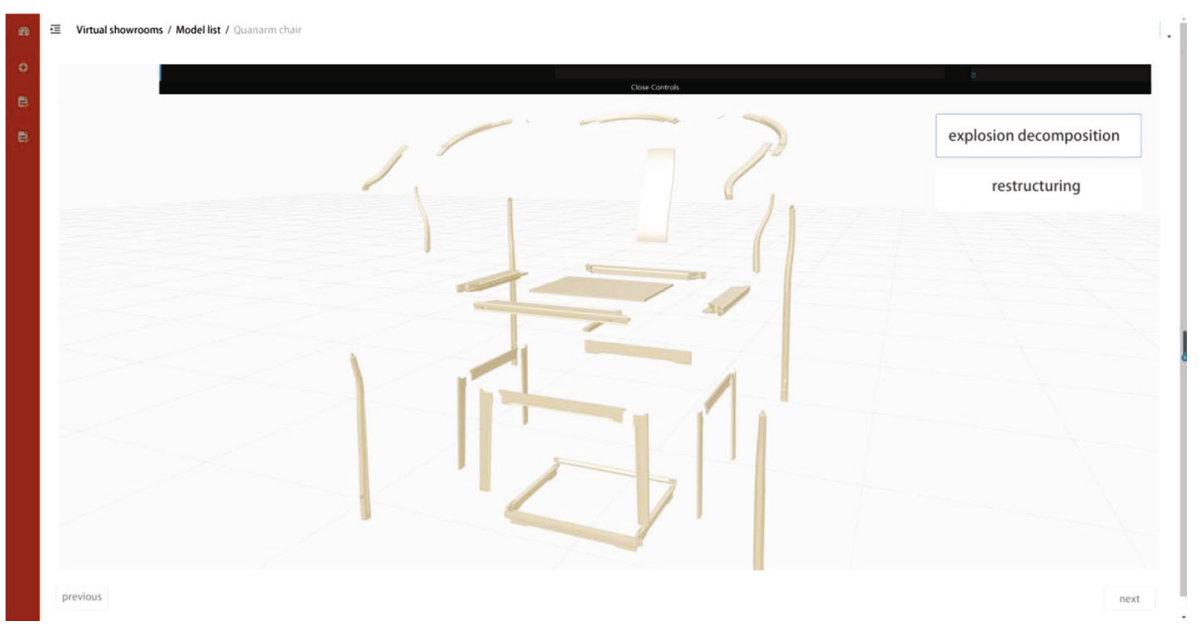

Figure 2: Virtual showroom plate.

tenon is just like the design idea of Kong Mingsuo and how to open and buckle is the design principle of mortise and tenon joint. The 3D modeling of mortise and tenon structure is also completed in each core module preset by the system, and the model is inserted into the shape selection link directly through the code.

Renderings and 3D models: at present, the generation of experimental results is completed by means of result simulation; that is, all the permutation and combination results are judged and calculated in advance, and their renderings and
3D models are placed in the program code in advance. Taking the chair innovative design experiment as an example, at present, more than 3,000 preset innovative forms have been calculated from 6 core modules. And along with the increase of options in each component module, the number of dimensional results will be generated (Figure 4).

On the whole, the logic of morphological innovation is to combine different morphological modules according to the building steps: collecting the modular forms of the same parts of furniture of the same shape, screening and inducing 


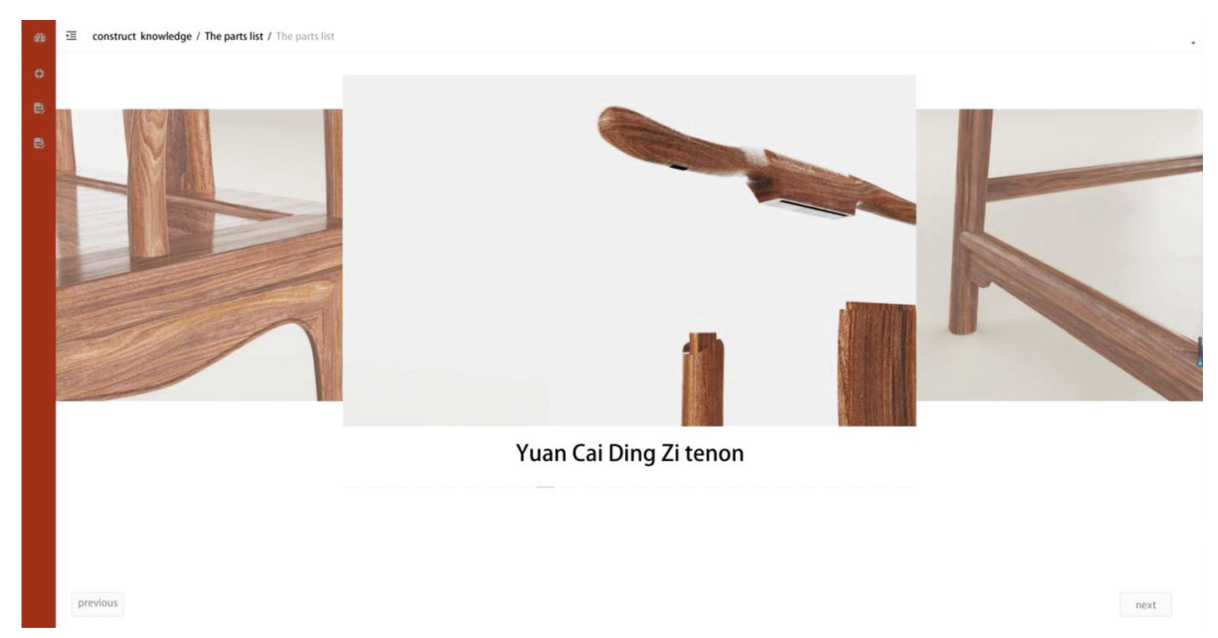

Figure 3: Construct knowledge plate.

the parts options, and then selecting the shapes of furniture parts according to steps. After different selections, arrangements, and combinations, more and more new forms can be formed. These forms conform to the internal modeling clues of Ming furniture. Morphology argues that modal design as a design thinking and design style should establish a deeper interaction between the object and the user as a certain design requirement [10]. Interactive experiments enable everyone to set up according to their own aesthetic requirements and finally create unique Ming furniture that conforms to the standard.

3.2.1. Scoring Method. In the scoring, the first part of "Virtual Showroom Learning" is to learn and observe the basic knowledge in order to respond to the interactive questions in the subsequent part, not involving experimental questions, but requiring students to use the content and interactive buttons of each showroom. In addition, a basic reading time was set in each furniture hall to monitor the students' observation of all the contents, and points would be deducted for omissions and insufficient time.

The second part, "construction knowledge learning", has several experimental projects, and each experiment is performed step by step. If there is a wrong operation, the system will prompt the error and re-experiment until the correct operation and completion of all steps. Each experiment will deduct the corresponding points for each wrong answer.

The third part, "Innovative Design Experiment", gives students a visual representation of the design results, and the results are scored collectively by the instructor and other students, which are scored in a subjective manner. The fourth part, "3D printing output", is given as an extra point. The final score of all four sections was combined to form each student's grade.

Comparative observations of experimental data from each student's four parts will be made, with the focus on the second part of the phenomenon. The difference of scores in the second part will show the difference of mastery of construction principles and order among students, clarify the difficulty of teaching, and give suggestions to students for follow-up. The data of the third part can show the popularity of the form and get the ranking of favorability based on the big data.

\section{Technical Architecture}

4.1. Technical Architecture. The whole experimental system architecture takes cloud storage and cloud computing as the core and restores the virtual reality data of each furniture and components to the web page according to the original proportion, which is convenient for end users to experience the real and natural building scenes and visual effects. We combine the prepared basic components and event system to build the system architecture diagram as shown in the following figure (Figure 5).

4.2. R\&D Technology. In order to realize the development of computer educational software and the construction of its application platform based on virtual simulation technology for the protection and activation design of Ming furniture, necessary computer hardware facilities and appropriate application programs must be used. At the same time, with the support of high-quality visual design, the experience quality of user experimental operation is realized.

First of all, hardware equipment is the foundation and guarantee.

Including cloud server and network requirements and conditions, the processor selects 16-core Intel processor to ensure that a large number of $3 \mathrm{D}$ models can rotate and scale smoothly during rendering. Compared with the conventional $16 \mathrm{G}$ running memory, this project adopts a higher version of $32 \mathrm{G}$ to ensure that the response time of users is short enough when operating and answering questions, and there will be no frame drop. The bandwidth is $100 \mathrm{MB}$ downlink bandwidth, which ensures that when multiusers need to use it in teaching scenes, there will be no server jamming and slow page loading;

Running environment of equipment terminal: for users, mainly teachers and students, there may be two categories of operating systems: Microsoft Windows and MAC Os, and corresponding system adaptations have been made, respectively. In addition, considering that the user's browser 

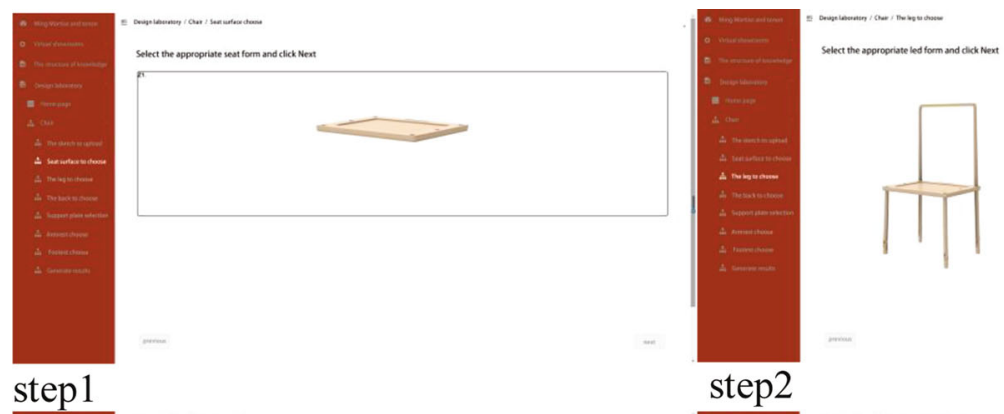

\section{step2}
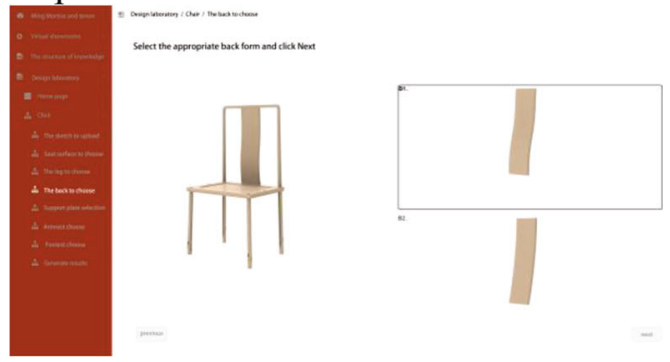

Step3
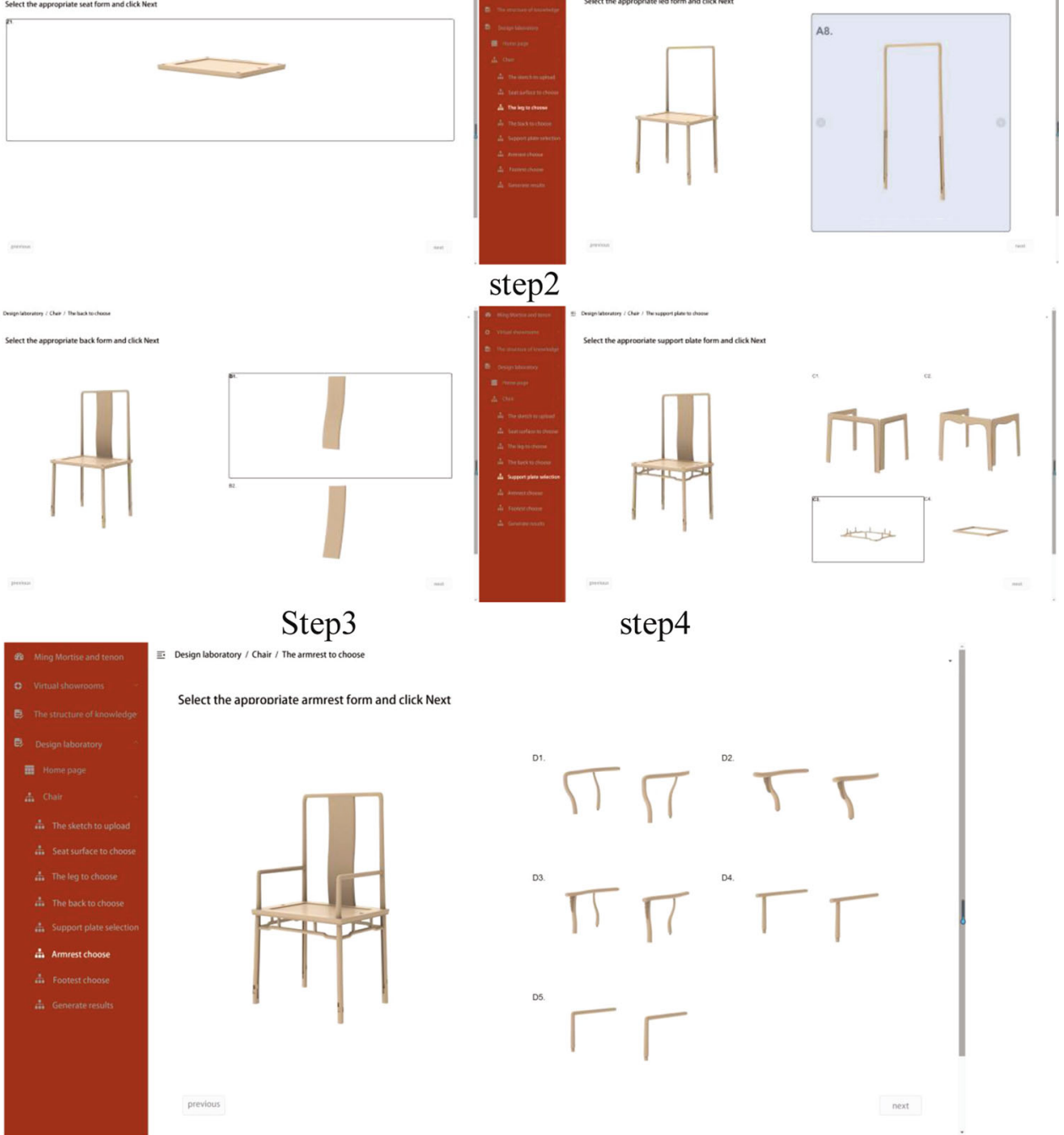

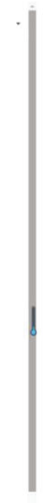
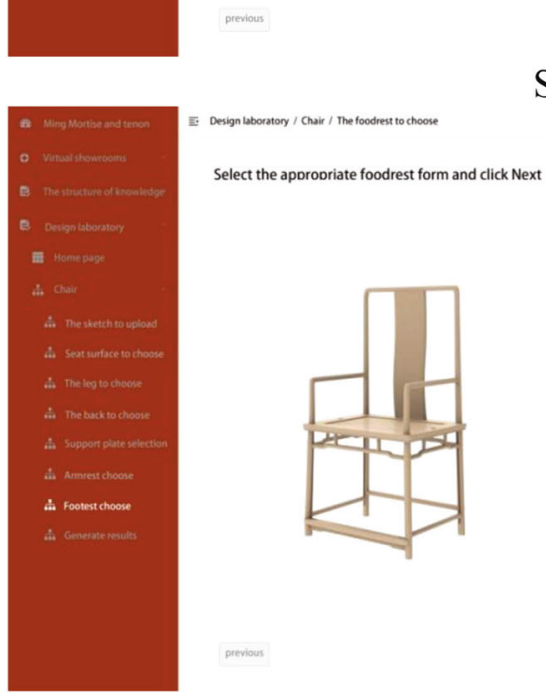

\section{Step5}

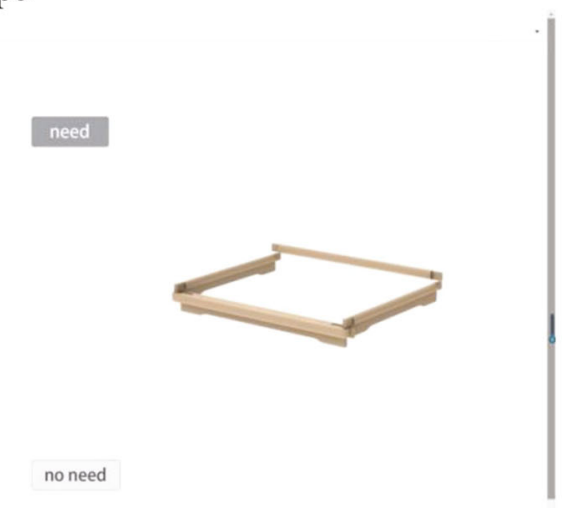

\section{Step6}

FIgURE 4: Innovative experimental plate operation step 1, step 2, step 3, step 4, step 5, and step 6.

also has inertia, the browsers such as Chrome, Firefox and 360 , which are more commonly used in the market, have also been adapted to ensure the smooth running environment of the terminal.
Cloud service guarantee of experimental quality: in order to improve the efficiency of users' learning courses, some modules adopt error feedback mechanism without reducing the quality of courses, so as to help users achieve 


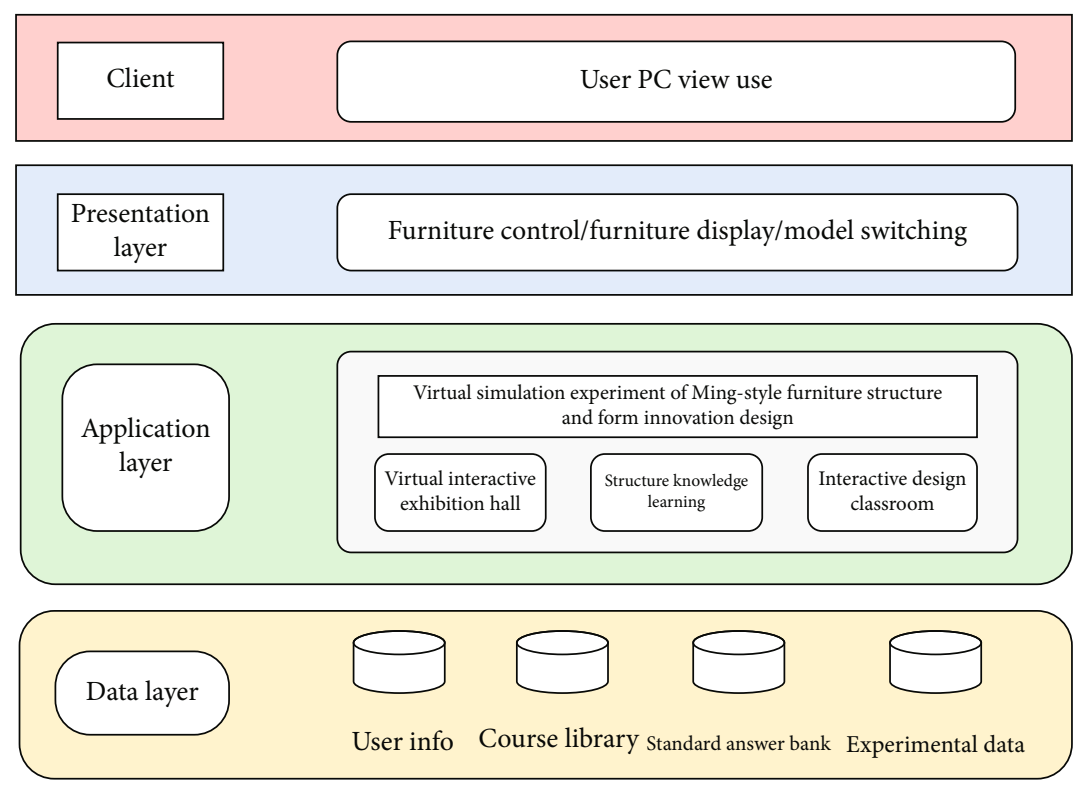

Figure 5: System architecture diagram and brief description.

the level of smoothly continuing the next stage of learning. In addition, the course scoring mechanism has been optimized to some extent, and scores are given according to the distribution method of each plate's weight, so as to ensure that the final generated scores can maximize the user's mastery.

4.3. Software Technology. Another part, development technology and tools are the means and quality of website content realization. The basic content and story system based on virtual reality expression on the platform should be realized by tool software. The expression of virtual reality of Ming furniture entity is the basic content and focus of this course teaching resources. It includes static model and dynamic interaction. In the planned three "story systems," specific simulation effects are given according to the experimental needs. In order to realize this current overall tool, software division of labor is as follows:

Reverse data acquisition: in order to achieve the accuracy of three-dimensional data model, we use MetraSCAN3DBLACK 3D scanning equipment and match the best VXelements9.0 application software to carry out threedimensional scanning to obtain the three-dimensional data of furniture solid objects. The highest scanning accuracy of the system is $0.025 \mathrm{~mm}$, and the precision volume accuracy is $0.064 \mathrm{~mm}$, thus realizing "absolute restoration" of basic data. Then, the model editing system of VXelements 9.0 is used to optimize the data, and then it is converted into an editable general 3D software file format.

Data reconstruction: after VXelements9.0 transcoding into RHINO3D editable files, according to the original model data, the application of RHINO3D modeling software to complete the virtual model reconstruction of the entity, this link includes exterior surface reconstruction and interior structure modeling, thus completing the complete data of furniture modeling and interior structure.
Visual Rendering and Dynamic Interaction. Using the rendering function of $3 \mathrm{D}$ Studio Max to realize the simulation imaging of static picture, for the convenience of operation and observation in all aspects of the experiment, the effects under each story system are designed differently. Use the white mold state in the virtual exhibition hall. Use the combination of white mold and real material in the interactive link of experiment. In order to achieve the effect of "visible is obtained" in the innovation link, the real material effect is adopted.

Blender realizes the dynamic interaction of furniture materials. Furniture model material for secondary mapping, further rendering, ensures the resulting $3 \mathrm{D}$ model on the page rendering effect as much as possible in line with the original state. Ensure smooth loading while ensuring high resolution. In the project, the video showing the independent mortise and tenon disassembly structure and the animation showing the components in the second half are completed by rhinoceros 3D modeling software, which can realize the dynamic process of zoom-in, zoom-out, rotational disassembly, and combination of components.

Sketch Up, HTML5, achieves two-dimensional animation generation. In order to reduce the overall load of the system and accelerate the speed of page reflection, we selectively change some links into two-dimensional animation instead of excessive interaction and accelerate the experimental process.

Visual Studio aims at the construction of immersive experience of VR virtual reality. In the experimental system, we plan to design two operating environments. For online operation, relying on the network environment, online experiments can be completed only by equipped with computers and broadband equipment with performance requirements. This platform is aimed at a wide range of learners. The other is VR virtual reality immersive experience environment. Completed in the virtual simulation laboratory at 
school, VR glasses are VIVE of HTC, and the equipment built in cooperation with Valve manufacturers in the world can meet the immersive experience requirements of users in all directions and truly realize "immersive" and "what you see is what you get."

Website construction: the website construction of this place mainly includes several main tasks: applying for domain name, applying for space, analyzing website function and demand layout, and designing website style. The application software responsible for the visual design of the website learning terminal is illustrate plane software, Visual Studio Code programming software is used for website construction, and Vue is used for programming framework. Three.js is used for generation, rendering and dynamic composition and decomposition of 3D components, microservice architecture is used for website deployment, Nginx agent and Docker deployment container are used, and Final Shell is used for remote server access visualization application software.

The morphological structure of Ming furniture has systematic and diverse content characteristics; so, the specific operation involves a huge amount of data. For example, in the first phase, aiming at the model observation and experimental design of ten pieces of furniture, there are more than 3,000 static renderings, more than 100 effective twodimensional drawings and millions of dynamic interactive codes. This experiment at this stage focuses on the understanding of the logical relationship between the form and construction of Ming furniture, and does not discuss the aesthetic form and aesthetic proportions of the local components, and therefore does not yet involve the regulation of the specific detailed parameters of the model components. As the study progresses, it can be extended to these contents in the future.

Now, it is made into the corresponding table as follows, so as to explain the classification more clearly (Table 1).

4.4. Platform Deployment. Developers first simulate user login in the test environment and carry out a series of stress tests, bug fixes, and other operations to ensure that there will be no root problems after the project goes online. For the subsequent requirements change test, an automated code deployment program is developed to ensure that the relevant requirements can be updated to the platform for users to use as soon as possible after being solved. In addition, the platform also makes relevant interface docking in the iLab experimental space, so that user data, such as operation time, operation score, step score, and other data, can be submitted to the iLab experimental platform at the same time when the final class is completed, which is convenient for teachers to view and count.

\section{Innovation Points of Project Application}

The virtual simulation experimental teaching of Ming-style furniture makes up for the previous problems of lack of physical teaching resources, high experimental cost, and long period. More importantly, the computer education software development and its application platform research based on the design of Ming furniture conservation and revitalization under virtual simulation technology are original. This greatly improves the students' learning motivation and efficiency, and the innovations are summarized as follows.

5.1. Innovation of Experimental Scheme Design Ideas. The traditional mortise and tenon intelligent system inheritance and design activation teaching construction fully embodies the principle of "combining reality with reality, complementing each other, and being real." Adopt virtual technology, break through the traditional experimental mode and time and space barrier, connect virtual and real, and comprehensively improve learners' interest in basic theory, innovative spirit, and participation [11].

Through the combination of virtual and real simulation, the virtual experimental operation and traditional experimental experience are combined, which transcends the skill display at the woodworking operation level and promotes the grasp and application of the Ming furniture knot concept.

In the design activation stage, the user-centered design concept can achieve the training goal of rapid design and rapid evaluation through the rapid reflection of module combination.

5.2. Innovation of Computer Intelligent Design and Application. Based on digital technology, this paper connects traditional creation with computer language. The operation mode of traditional furniture structure is realized by digital intelligent algorithm, and a set of design logic of mortise and tenon furniture structure, and form is tried to be constructed. It is an unprecedented achievement, a pioneering work in the field of traditional furniture design and manufacturing, and an important attempt of computer intelligence in the revival of traditional cultural industry.

Using 3D scanning reverse data acquisition technology and intelligent operation of computer engine, big data simulation is realized, and the design software of Ming furniture is developed. Interpreting and presenting the structural thinking and design concept of Ming furniture by means of computer information technology and transforming it into design program software can greatly reduce the design time cost and financial cost, which is conducive to largescale popularization.

5.3. Innovation of Teaching Information Technology. The use of virtual technology expands the protection and educational resources of Ming furniture, makes the teaching and communication counseling mode of students more diversified and flexible, has enough immersive experience, enhances the sense of participation in experimental teaching, and strongly stimulates interest. Open sharing is realized through Internet technology, which effectively expands the communication and influence of Ming furniture, and better combines protection, education, activation, and innovation. The combination mechanism of production, education, and research, which connects with enterprises, institutions, 
TABLE 1: Software and hardware support.

\begin{tabular}{|c|c|}
\hline Development technology & 3D simulation, 2D animation, HTML5, VR \\
\hline Development tools & 3D Studio Max, SketchUp, Blender, Visual Studio, Illustrate \\
\hline Running environment & $\begin{array}{c}\text { Server: CPU } 8 \text { cores, memory } 8 \text { GB, disk } 50 \text { GB, } 8 \text { GB video memory, GPU model GTX2060 } \\
\text { Operating system: Windows server version: Windows } 10 \\
\text { Database: Mysql }\end{array}$ \\
\hline Experimental quality & $\begin{array}{l}\text { Number of single scene model faces: one; mapping resolution: } 512 \mathrm{px} \times 512 \mathrm{px} \text {; number of } \\
\text { renderings per frame: } 80 \text { calls; action feedback time: Ms; display refresh rate: } 60 \mathrm{FPS} \text {; resolution: } \\
1920 \mathrm{ppi} \times 1080 \mathrm{ppi}\end{array}$ \\
\hline $\begin{array}{l}\text { Cloud server and network } \\
\text { requirements and conditions }\end{array}$ & $\begin{array}{l}\text { The uplink and downlink bandwidth is over } 50 \mathrm{M} \text {. through testing, when the bandwidth is over } \\
\qquad 100 \mathrm{M} \text {, it can have faster loading speed and better interactive experience. }\end{array}$ \\
\hline
\end{tabular}

and industries, enriches the depth and breadth of learning content.

\subsection{Extension and Expansion of Traditional Teaching. Open} sharing, school-enterprise cooperation and collaborative innovation have established a sustainable virtual experimental teaching support system. Completed the construction of network sharing platform, online simulation, experiment reservation, online guidance, and virtual environment of resource sharing and can open and share operation experiments online and offline.

\section{Conclusion}

Using cutting-edge scientific and technological achievements in cultural innovation can create new species projects which are different from traditional modeling design. The research of this education platform not only has the whole product intellectual innovation from culture to product, from creativity to ecology, but also constructs the bottom logic of talent training, technology management and production management that can open up the innovation industry of Ming furniture.

Even it can lead to new ideas, new technologies, and new business models. In the future research, there will be more elements interwoven, and the process will also uphold the open exploration and the integration of ideas and cutting-edge technologies. Future research will have more elements of interweaving, which is realized by docking CNC technology on the basis of virtual data, and needs to continue to deepen the benefits of artificial intelligence algorithms and cloud computing to achieve the design side and the output side of the integrated one-stop service. Through the Internet of things to achieve the interconnection of the process of precise control, always adhering to the openness of exploration and the integration of ideas and cutting-edge technology, in the future to intelligent manufacturing to reach the organic renewal of traditional culture.

\section{Data Availability}

No data were used to support this study.

\section{Conflicts of Interest}

The authors declare that there are no conflicts of interest regarding the publication of this article.

\section{References}

[1] Y. Yao, Ming-style furniture research, China Construction Industry Press, 2002.

[2] N. Noah and S. Das, "Exploring evolution of augmented and virtual reality education space in 2020 through systematic literature review," Computer Animation and Virtual Worlds, vol. 32, no. 3-4, p. 2021, 2020.

[3] A. P. Murillo and A. Yoon, "a study of emerging trends in digital preservation literature: an analysis of journal articles presented in course syllabi," Journal of Librarianship and Information Science, vol. 53, no. 4, pp. 615-629, 2021.

[4] S. Weiwei, L. Dejun, and L. Jiyao, "Exploration of new Chinese furniture design based on semiotics theory," Journal of Northwest Forestry University, vol. 6, pp. 169-173, 2013.

[5] Y. Dehua, "Talking about the mortise and tenon of traditional Chinese furniture," Art and Design, vol. 34, no. 6, pp. 96-98, 2007.

[6] W. Shixiang, Ming-Style Furniture Research, Sanlian Bookstore Co., Ltd, Hong Kong, 2002.

[7] J. X. Han, M. Y. Ma, and K. Wang, "Product modeling design based on genetic algorithm and BP neural network," Neural Computing and Applications, vol. 33, no. 9, pp. 4111-4117, 2021.

[8] S. al Awadhi, N. al Habib, D. al-Murad et al., "Interactive virtual reality educational application," Advances in Science, Technology and Engineering Systems, vol. 3, no. 4, pp. 72-82, 2018.

[9] M. L. Alan, "Book review: KJ Varnum (ed.),Beyond reality: augmented, virtual, and mixed reality in the library," Journal of Librarianship and Information Science, vol. 53, no. 4, pp. 709-710, 2021.

[10] M. Gunduz and T. Yetisir, “A design reuse technology to increase productivity through automated corporate memory system," Neural Computing and Applications, vol. 29, no. 9, pp. 609-617, 2018.

[11] L. Gao, B. Wan, G. Liu, G. Xie, J. Huang, and G. Meng, "Investigating the effectiveness of virtual reality for culture learning," International Journal of Human-Computer Interaction, vol. 37, no. 18 , pp. 1771-1781, 2021. 